Table 1. Comparison of the total FLARE-RA scores (13q) between the disease activity groups (DAS28CRP)

\begin{tabular}{lcccccc}
\hline & & Remission & LDA & MDA & HDA & P-value \\
\hline BL & Patients (n) & 62 & 3 & 1 & 0 & \\
& FLARE Q & $1.8 \pm 0.8$ & $1.5 \pm 0.3$ & 1.3 & & 0.800 \\
M3 & Patients (n) & 50 & 11 & 5 & 0 & \\
& FLARE Q & $2.1 \pm 1.0$ & $3.0 \pm 0.9$ & $3.5 \pm 1.4$ & & 0.004 \\
M6 & Patients (n) & 52 & 5 & 9 & 0 & \\
& FLARE Q & $2.1 \pm 0.8$ & $3.1 \pm 1.3$ & $3.1 \pm 1.9$ & & 0.057 \\
M9 & Patients (n) & 48 & 10 & 7 & 1 & \\
& FLARE Q & $2.1 \pm 0.9$ & $2.8 \pm 1.1$ & $3.3 \pm 1.6$ & 2.4 & 0.079 \\
M12 & Patients (n) & 52 & 8 & 6 & 0 & \\
& FLARE Q & $2.1 \pm 1.0$ & $3.1 \pm 0.8$ & $3.2 \pm 1.0$ & & 0.002 \\
& & & & & & \\
\hline
\end{tabular}

Conclusion: The FLARE-RA scores seem to reliably discern between patients with and without an OMERACT flare. A cut-off of 2.7 on the current questionnaire (r11q) had the optimal sensitivity and specificity to identify an OMERACT flare. Disclosure of Interests: Delphine Bertrand: None declared, Veerle Stouten: None declared, Sofia Pazmino: None declared, Diederik De Cock: None declared, Anneleen Moeyersoons: None declared, Rene Westhovens Grant/research support from: Celltrion Inc, Galapagos, Gilead, Consultant of: Celltrion Inc, Galapagos, Gilead, Speakers bureau: Celltrion Inc, Galapagos, Gilead, Johan Joly: None declared, Patrick Verschueren Grant/research support from: Pfizer unrestricted chair of early RA research, Speakers bureau: various companies DOI: 10.1136/annrheumdis-2020-eular.2832

\section{FRI0567 CONSTRUCT VALIDATION OF PROMIS SHORT FORM AND PROFILE-29 T-SCORES WITH SF-36 IN RHEUMATOID ARTHRITIS PATIENTS TREATED FOR YEAR: RESULTS FROM A REAL-WORLD EVIDENCE- BASED STUDY IN THE UNITED STATES}

C. Bingham ${ }^{1}$, S. Kafka ${ }^{2}$, S. Black ${ }^{2}$, S. Xu ${ }^{3}$, W. Langholff' ${ }^{2}$, J. Curtis ${ }^{4}{ }^{1}$ John Hopkins Arthritis Center, Baltimore, MD, United States of America; ${ }^{2}$ Janssen Scientific Affairs, LLC., Horsham, PA, United States of America; ${ }^{3}$ Janssen Research \& Development, LLC, Spring House, PA, United States of America; ${ }^{4} U$ of Alabama at Birmingham, Dept of Medicine, Birmingham, AL, United States of America

Background: Use of patient-reported outcomes (PROs) to assess health-related quality of life in clinical practice, research studies, and clinical trials in rheumatoid arthritis (RA) remains an ongoing area of research. SF-36 is commonly used in RA trials but is not feasible for routine use in clinical practice settings. The Patient Reported Outcomes Measurement Information System (PROMIS) may address this gap but has not been widely assessed in RA patients starting therapy in a real-world comparative effectiveness study, nor examined in that setting in relation to the SF36 and Clinical Disease Activity Index (CDAI).

Objectives: To assess validity of PROMIS based on Comparative and Pragmatic Study of Golimumab Intravenous (IV) Versus Infliximab in Rheumatoid Arthritis (AWARE), an ongoing Phase 4 study providing real-world assessment of IV tumor necrosis factor inhibitor (TNFi) medications in RA patients.

Methods: AWARE is a prospective, non-interventional, 3-year study conducted at 88 US sites. RA patients were enrolled when initiating TNFi treatment. Treatment decisions were made by treating rheumatologists. We report baseline PROMIS-29 (7 domains and pain intensity), PROMIS Pain Interference (PI) Short Form (SF) $6 \mathrm{~b}$ (PI6b) and PROMIS Fatigue (F) Short Form 7a (F7a), domain T-Scores, and SF-36 subdomain and Component Scores (CS) in AWARE patients. Here we report baseline data obtained from the final 1-year AWARE dataset. Correlations between PROMIS measures and comparable SF-36 component scores were calculated using Pearson correlations. Data is shown as mean \pm standard deviation (SD).

Results: At baseline, mean CDAl of all patients $(n=1262)$ was $32.3 \pm 15.6$, with $70.4 \%$ in high disease activity (HDA, CDAl>22), $22.8 \%$ in moderate disease activity (MDA, CDAl: $>10$ and $\leq 22$ ), $6.1 \%$ in low disease activity (LDA, CDAl: $>2.8$ and $\leq 10$ ), and $0.7 \%$ in remission (CDAl $\leq 2.8$ ). Mean PROMIS scores were $>0.5 \mathrm{SD}$ worse than population means for Physical Function (PF, 38.1 \pm 6.84$)$, PI (63.4 \pm 7.68 ), F (58.8 \pm 9.95$)$, Sleep Disturbance $(55.1 \pm 8.68)$; and Ability to Participate in Social Roles/Activities (PSRA, 43.4 \pm 8.58 ). Baseline Depression and Anxiety were within 0.5 SD of population T-scores. PI6b, F7a, and P29 domain T-scores correlated with the comparable SF-36 subdomain and component scores ( $r$ 's $>0.58$ ), except sleep for which no comparable SF-36 element was applicable. Examples include: $\mathrm{P} 6 \mathrm{~b}(\mathrm{r}=-$ $0.80)$ and P29-PI (0.81) with SF-36 Bodily Pain; F7a (-0.77) and P29-F (-0.77) with SF-36 Vitality; P29-PF with SF-36 PF (0.77), Role-Physical (0.69), and Physical CS (0.73); P29 Anxiety with SF-36 Mental Health (-0.72), Role-Emotional (-0.56), Mental CS (-0.70); and P29-PRSA with SF-36-Social Functioning (0.71). Mean PROMIS-29 T-scores (except Anxiety and Sleep Disturbance) among patients with HDA were significantly different from patients with MDA, LDA or remission $(p<0.001$ for all).
Further, mean PROMIS T-scores of PF, F, PSRA, PI, Pain Intensity, PI6b and P7a among patients with MDA were significantly different from patients with more or less active RA (by CDAl category).

Conclusion: Analysis of baseline results from a large cohort of RA patients indicates high correlations between individual P29 domain T-scores and SF-36 component scores, as well as categorical CDAI, providing strong evidence of PROMIS construct validity in a real-world population of RA patients.

Disclosure of Interests: Clifton Bingham Grant/research support from: Bristol-Myers Squibb, Consultant of: Bristol-Myers Squibb, Shelly Kafka Employee of: Janssen Scientific Affairs, LLC, Shawn Black Employee of: Janssen Research \& Development, LLC, Janssen Scientific Affairs, LLC, Stephen Xu Employee of: Janssen Research \& Development, LLC, Wayne Langholff Employee of: Janssen Research \& Development, LLC, Jeffrey Curtis Grant/research support from: AbbVie, Amgen, Bristol-Myers Squibb, Corrona, Janssen, Lilly, Myriad, Pfizer, Regeneron, Roche, UCB, Consultant of: AbbVie, Amgen, Bristol-Myers Squibb, Corrona, Janssen, Lilly, Myriad, Pfizer, Regeneron, Roche, UCB

DOI: 10.1136/annrheumdis-2020-eular.2255

\section{FRI0568 \\ MEASURING DAILY PHYSICAL ACTIVITY IN AXSPA PATIENTS: CONTENT VALIDITY AND MEASUREMENT PROPERTIES OF THE NEW AXSPA-SQUASH}

M. Carbo ${ }^{1}$, D. Paap ${ }^{1}$, F. Maas ${ }^{1}$, A. J. Baron ${ }^{1}$, L. Van Overbeeke ${ }^{1}$, M. Siderius ${ }^{1}$, F. Wink ${ }^{2}$, H. Bootsma ${ }^{1}$, S. Arends ${ }^{1}$, A. Spoorenberg ${ }^{1}{ }^{1}$ University Medical Center Groningen, Rheumatology and Clinical Immunology, Groningen, Netherlands; ${ }^{2}$ Medical Center Leeuwarden, Rheumatology, Leeuwarden, Netherlands

Background: The ASAS-EULAR recommendations for management of axial Spondyloarthritis (axSpA) includes that patients should be encouraged to exercise. ${ }^{1}$ So far, there is no validated instrument for measuring daily physical activity in axSpA. Our previous study recommends to adapt the Short QUestionnaire to Assess Health-enhancing physical activity (SQUASH) to improve the validity in axSpA patients. ${ }^{2}$

Objectives: AxSpA-disease specific adaptation of the physical activity questionnaire SQUASH to improve content validity and measurement properties.

Methods: This study was conducted according to the OMERACT-filter within the Groningen Leeuwarden AxSpA (GLAS) cohort and was performed in two parts. Part 1: adaptation and evaluation of content validity using a qualitative stepwise approach with in-depth interviews with different healthcare professionals $(n=9)$ and patients $(n=8)$, field testing in patients $(n=10)$, and consensus meeting for final adaptations. Thereafter, content validity $(n=45)$ was tested by filling out axSpA-SQUASH and SQUASH in random order two weeks apart. Part 2: measurement properties were tested using the International Physical Activity Questionnaire (IPAQ) as comparator Criterion validity $(n=40)$ : Spearman's correlation with accelerometer as golden standard and classification accuracy of intensity. Construct validity $(n=106)$ : Spearman's correlation with disease activity, physical functioning and quality of life as clinical outcome with expected fair to moderated associations. Test-retest reliability $(n=45)$ : intraclass correlation coefficients (ICC) after 2 weeks. Responsiveness $(n=47)$ : standardized response mean (SRM) after 3 months stratified by Ancor method. Results: In total 156 patients were included: mean age $48 \pm 13$ years, $56 \%$ males, $72 \%$ HLA-B27 positive, symptom duration $21 \pm 13.3$ years and ASDAS $2.0 \pm 1.0$. Part 1: main adaptations were better explanation of intensities, adding answer option "not applicable", examples were modernized, physiotherapy and activity "shopping" were added. Compared to the original SQUASH, the adapted axSpA-SQUASH measured a systematically higher activity count and had less missing values $(8 \%$ vs. $32 \%)$. Part 2: criterion validity: axSpA-SQUASH correlated better with acceler ometer compared to IPAQ ( $\rho=0.51$ vs. $\rho=0.35$ ). Classification accuracy: accelerometer defined most activity as light (97\%), whereas axSpA-SQUASH and IPAQ defined most activity as moderate intensity (55\% and $62 \%$ resp.). Construct validity: correlations were low to moderate and strongest for axSpA-SQUASH compared to IPAQ. Construct validity: correlations were low to moderate and stronger for axSpASQUASH compared to IPAQ (BASDAI -0.27 vs -0.15 , BASDAI -0.27 vs. -0.15 ASDAS -0.24 vs -0.09 , BASFI -0.39vs. -0.21 , ASQoL -0.39 vs. -0.35 ). Test-retest reliability: ICC axSpA-SQUASH: 0.80. Responsiveness: axSpA-SQUASH changed over time in the corresponding direction (Table 1). Feasibility: considered comprehensible and average completion time was 7 minutes.

Table 1. Responsiveness of the axSpA-SQUASH versus change in BASDAI

\begin{tabular}{lcccc}
\hline & SRM & $95 \% \mathrm{Cl}$ & BASDAI T1 & BASDAI T2 \\
\hline Improved $(\mathrm{n}=12)$ & -0.36 & -0.99 to 0.28 & $5.01(2.10)$ & $3.93(1.60)$ \\
Stable $(\mathrm{n}=21)$ & 0.28 & -0.18 to 0.73 & $3.76(2.05)$ & $3.76(2.05)$ \\
Decreased $(\mathrm{n}=14)$ & 0.75 & 0.18 to 1.33 & $4.71(1.96)$ & $5.79(2.42)$ \\
\hline
\end{tabular}

